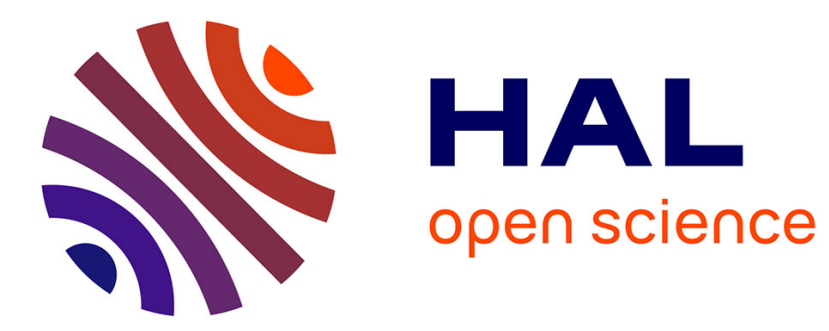

\title{
Manifestation of Hamiltonian monodromy in nonlinear wave systems
}

\author{
E. Assemat, Christine Michel, Antonio Picozzi, Hans-Rudolf Jauslin, \\ Dominique Sugny
}

\section{- To cite this version:}

E. Assemat, Christine Michel, Antonio Picozzi, Hans-Rudolf Jauslin, Dominique Sugny. Manifestation of Hamiltonian monodromy in nonlinear wave systems. Physical Review Letters, 2011, 106, pp.014101. 10.1103/PhysRevLett.106.014101 . hal-00699917

\section{HAL Id: hal-00699917 https://hal.science/hal-00699917}

Submitted on 22 May 2012

HAL is a multi-disciplinary open access archive for the deposit and dissemination of scientific research documents, whether they are published or not. The documents may come from teaching and research institutions in France or abroad, or from public or private research centers.
L'archive ouverte pluridisciplinaire HAL, est destinée au dépôt et à la diffusion de documents scientifiques de niveau recherche, publiés ou non, émanant des établissements d'enseignement et de recherche français ou étrangers, des laboratoires publics ou privés. 


\title{
Manifestation of Hamiltonian Monodromy in Nonlinear Wave Systems
}

\author{
E. Assémat, C. Michel, A. Picozzi, H. R. Jauslin, and D. Sugny* \\ Laboratoire Interdisciplinaire Carnot de Bourgogne (ICB), UMR 5209 CNRS-Université de Bourgogne, \\ 9 Av. A. Savary, BP 47 870, F-21078 DIJON Cedex, France
}

(Received 24 September 2010; published 5 January 2011)

\begin{abstract}
We show that the concept of dynamical monodromy plays a natural fundamental role in the spatiotemporal dynamics of counterpropagating nonlinear wave systems. By means of an adiabatic change of the boundary conditions imposed to the wave system, we show that Hamiltonian monodromy manifests itself through the spontaneous formation of a topological phase singularity ( $2 \pi$ - or $\pi$-phase defect) in the nonlinear waves. This manifestation of dynamical Hamiltonian monodromy is illustrated by generic nonlinear wave models. In particular, we predict that its measurement can be realized in a direct way in the framework of a nonlinear optics experiment.
\end{abstract}

DOI: 10.1103/PhysRevLett.106.014101

PACS numbers: 05.45.- a, 02.30.Ik, 45.05.+x

Introduction.-The geometric analysis of complex dynamical systems is known to provide valuable physical insight, in particular with regard to the robustness of a physical phenomenon identified empirically. An illustrative example of the richness of this geometrical qualitative approach is provided by the Berry phase [1], which generated an immense interest throughout different fields of physics and quantum chemistry. Another important example is provided by the concept of Hamiltonian monodromy. It may be regarded as the simplest topological obstruction to the existence of global action-angle variables in Hamiltonian integrable systems governed by ordinary differential equations (ODE) [2]. The quantum analogue of this concept was formulated in [3] and was the starting point of numerous studies of ODE relevant to both classical and quantum physics, which revealed that monodromy is a universal phenomenon that occurs in many different physical situations [4]. Our aim in this Letter is to show that the concept of monodromy finds a remarkable application in systems ruled by partial differential equations (PDE). We consider a system of counterpropagating nonlinear waves, which is in essence an infinite dimensional dynamical system. It was recently shown that this kind of PDE system exhibits a relaxation process toward a stationary state, which lies in the neighborhood of a singular torus associated with the corresponding ODE system $[5,6]$. Since singular tori are responsible for the existence of nontrivial monodromy in Hamiltonian ODE systems [2], the natural important question that arises from these works is the problem of the existence of Hamiltonian monodromy in PDE systems. This paper can be viewed as a first step in this new and open field of research.

Hamiltonian monodromy has been mainly used to characterize integrable physical systems from the static point of view. More recently, it has been proposed to extend this concept to a dynamical process in nonautonomous ODE systems, by introducing an abstract time-dependent perturbation in the system [7]. Here, we show that the dynamical concept of monodromy acquires a natural physical application in the framework of the spatiotemporal dynamics of PDE wave systems. Indeed, by means of an adiabatic change of the boundary conditions, the system goes through a series of stationary states whose corresponding projection in the energy-momentum diagram describes a closed loop. The numerical simulations of the PDE system reveal the spontaneous formation of a phase singularity (i.e., $2 \pi$ phase defect) in the waves when the loop encloses the singular torus, whereas no phase shift is observed for a trivial loop that does not enclose the singularity. We show that this phenomenon is a manifestation of the nontrivial Hamiltonian monodromy of the system. In particular, the evolution of the phase of the nonlinear wave possesses all the topological properties of Hamiltonian monodromy [2]. The numerical simulations of the PDE also reveal that the formation of the phase defect is a robust phenomenon of the spatiotemporal dynamics. Furthermore, we generalize our results to fractional Hamiltonian monodromy [8], which is characterized by the formation of a fractional $\pi$-phase singularity in the wave. Remarkably, in this case the wave does not recover its initial state when the system is subjected to a closed loop. Finally, we underline that the numerical simulations presented here correspond to a realistic nonlinear optical experiment, in which a manifestation of the nontrivial monodromy of the system could be measured in a direct way.

A PDE model.-As an illustrative example, we consider the nonlinear evolution of a wave in a periodic potential, a problem that finds applications in a variety of physical systems, ranging from optical waves in nonlinear gratings, electrons in crystals, or periodically confined Bose-Einstein condensates $[9,10]$. We analyze the one-dimensional spatiotemporal dynamics of the nonlinear wave in the neighborhood of a forbidden frequency band gap,

$$
\begin{aligned}
& \frac{\partial u}{\partial t}+\frac{\partial u}{\partial z}=i \kappa v+i \gamma\left(|u|^{2}+2|v|^{2}\right) u, \\
& \frac{\partial v}{\partial t}-\frac{\partial v}{\partial z}=i \kappa u+i \gamma\left(|v|^{2}+2|u|^{2}\right) v,
\end{aligned}
$$


where $u$ and $v$ are the counterpropagating complex wave amplitudes, which originate in Bragg reflections of the nonlinear wave on the periodic potential. $\kappa$ and $\gamma$ are the linear and nonlinear coefficients, respectively. When the following boundary conditions $u(z=0, t)=u_{0}$ and $v(z=L, t)=v_{L}$ are imposed at the ends of the medium of length $L$, we observe, under rather general conditions, that the PDE system exhibits a relaxation process toward a stationary state [5]. The dynamics of the stationary solutions is governed by the Hamiltonian $H$

$$
H=-2 \kappa \sqrt{I_{u} I_{v}} \cos \left(\phi_{u}+\phi_{v}\right)-4 \gamma I_{u} I_{v}-\gamma\left(I_{u}^{2}+I_{v}^{2}\right)
$$

where we have introduced the real coordinates $\left(I_{u}, \phi_{u}, I_{v}\right.$, $\phi_{v}$ ) defined by $u=\sqrt{2 I_{u}} e^{i \phi_{u}}$ and $v=\sqrt{2 I_{v}} e^{-i \phi_{v}}$. The corresponding Hamiltonian system is integrable since the momentum $K=I_{u}-I_{v}$ is a constant of motion. The energy-momentum diagram $(H, K)$ of this system exhibits an isolated singular point at $H=K=0$ associated to a singular pinched torus [see Fig. 1]. It was shown in Ref. $[5,6]$ that the relaxation process is due to the presence of this singular torus and that the stationary state lies in a regular torus in the neighborhood of the singular one. Using general arguments about monodromy [2], one can show that the stationary system exhibits a nontrivial monodromy. Our objective is now to construct a dynamical spatiotemporal process that reveals the manifestation of this topological behavior.

Numerical simulations.- Starting from a given stationary state of the system and a fixed length $L$ of the medium, we change adiabatically the boundary conditions $I_{u}(0)$, $\phi_{u}(0), I_{v}(L)$, and $\phi_{v}(L)$. For sufficiently slow variations of these parameters, the spatiotemporal dynamics follows adiabatically the stationary states associated to the boundary conditions. We choose these conditions in such a way that the stationary system describes a loop in the energymomentum diagram $(H, K)$. We plotted schematically in Fig. 1 two examples of loops that we followed in the simulations by integrating numerically the PDE system (1). Figures 2 and 3 show the corresponding numerical

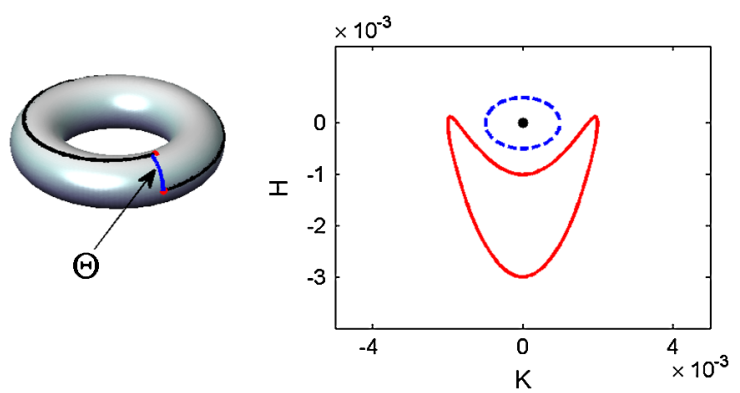

FIG. 1 (color online). (Left panel) Schematic illustration of the twist angle $\Theta$ on a regular torus. (Right panel) Zoom of the energy-momentum diagram near $H=K=0$ of the Hamiltonian system of Eq. (2). The full dot at $H=K=0$ indicates the position of the singular pinched torus. The trivial and nontrivial loops are denoted with a solid and a dashed lines, respectively. results obtained for a loop surrounding the singular point $H=K=0$, and a trivial loop that does not enclose this point. Note that the nontrivial loop cannot be smoothly deformed to avoid surrounding the singular point. In Fig. 2(a), each point of the diagram corresponds to the averages $\tilde{K}=\int_{0}^{L} K(z, t) d z / L$ and $\tilde{H}=\int_{0}^{L} H(z, t) d z / L$ of $H$ and $K$ over the length $L$ at times $t=n \tau(n=$ $0,1, \cdots, N)$, where $N$ is the number of points in the loop and $\tau$ is a fixed time interval. We have also plotted in Fig. 2(b) the evolution of the phase difference $\phi_{u}(L)-$ $\phi_{u}(0)$ as a function of time. We observe that the phase difference varies linearly with $n$ and acquires a $2 \pi$ shift over the course of the loop. This is in contrast with the simulation of the trivial loop, in which the phase difference $\phi_{u}(L)-\phi_{u}(0)$ returns back to its initial value once the loop is completed [see Fig. 3]. The mechanism underlying the formation of the $2 \pi$ phase singularity in the PDE system is reported in Fig. 4: At $n=75$ the modulus of the wave $u$ vanishes exactly at $z \simeq L / 2$, which thus permits the phase to exhibit the $2 \pi$ discontinuity. The field $u$ subsequently preserves the phase defect for $n>75$, until it recovers its initial state (modulo $2 \pi$ ) at $t=N \tau$ [see Fig. 4(a)]. Note that the field $v(z, t)$ exhibits an evolution similar to $u(z, t)$.

The simulations of the nontrivial loop have been realized for different values of the time $\tau$. For $\tau=500$, we are in the quasiadiabatic regime; i.e., the loop described by the spatiotemporal dynamics is very close to the ideal adiabatic loop, the average difference $|H-\tilde{H}|$ being lower than $10^{-5}$. As expected, the dynamics becomes more perturbed as the time $\tau$ decreases [see Fig. 2]. However, the remarkable result is that the PDE system still describes a loop around the singular point, even for times as small as
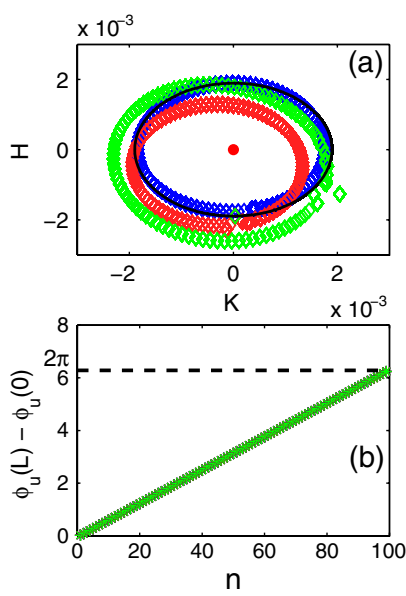

FIG. 2 (color online). (a) Different numerical loops in the energy-momentum diagram for different times $\tau=10$ [light grey (green)], 25 [grey (red)], 50 [dark grey (blue)], and 500 (black). (b) Evolution of the phase difference $\phi_{u}(L)-\phi_{u}(0)$ as a function of the coordinate $n$ for $N=100$ (see the text). Note that all plots for $\tau=10,25,50$, and 500 are superposed in (b). Parameters are $\kappa=1$ and $\gamma=0.2$. 


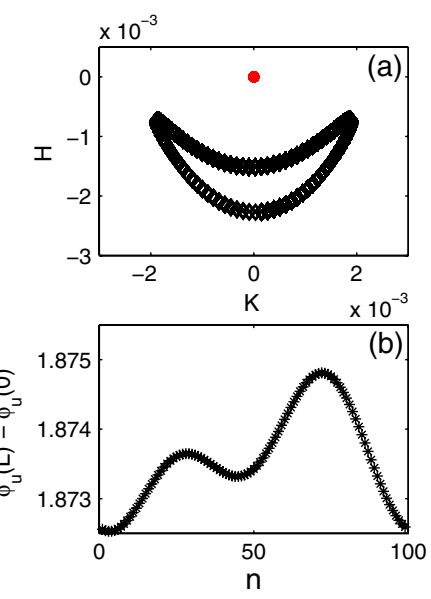

FIG. 3 (color online). Same as Fig. 2 but for a trivial loop in the $(H, K)$ diagram and a relaxation time $\tau=500$.

$\tau=10$ [Fig. 2(a)]. The robustness of this topological property becomes even more apparent through the analysis of the temporal evolution of the phase difference $\phi_{u}(L)-$ $\phi_{u}(0)$ reported in Fig. 2(b). It reveals that the phase shifts are independent of the values of the times $\tau$. This shows that the PDE space-time dynamics exhibits a topological behavior, which is close to the behavior of the adiabatic regime. We shall see in the following that these numerical observations constitute a signature of the nontrivial monodromy of the system.

Manifestation of nontrivial Hamiltonian monodromy.Hamiltonian monodromy is a topological property which is related to the change of the action-angle coordinates along a loop in the energy-momentum diagram. In its simplest form, for a two degree of freedom Hamiltonian system, the monodromy can be computed from the rotation number $\Theta$ and the first return "time" $Z$ [2] which are two functions of
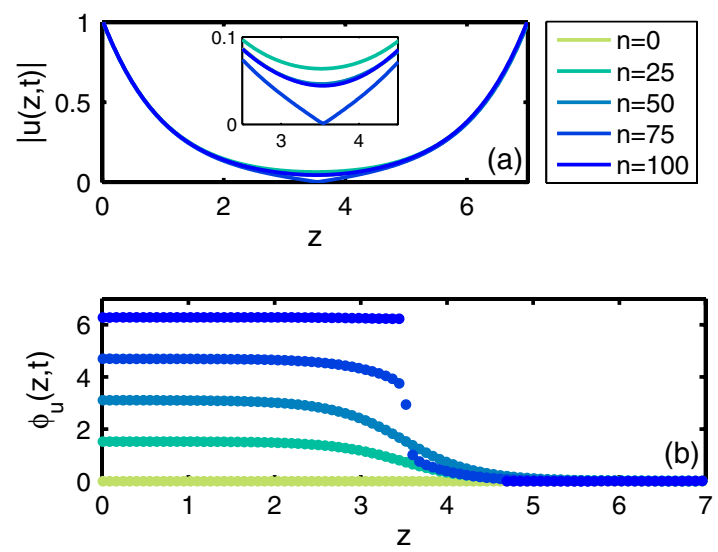

FIG. 4 (color online). Spatial profiles of the modulus $|u|(z, t)$ (a), and phase $\phi_{u}(z, t)(\mathrm{b})$, at different times $t$, corresponding to the simulation of Fig. 2 for $\tau=500$. The inset in (a) is a zoom of $|u|(z, t)$ near the value $|u| \simeq 0$. Note that $\phi_{u}(z, t=0)=$ $\phi_{u}(z, t=N \tau)[2 \pi]$, so that the initial and final states of the loop coincide.
$H$ and $K$ [we recall that the variable $z$ plays the role of an evolution time variable for the stationary ODE system (1)]. On a regular torus, the orbits of the momentum $K$ are circles parametrized by the angle $\theta$ conjugate to $K$. $Z$ is the time needed for an orbit of $H$ to reach the orbit of $K$ starting from a point of this orbit, while $\Theta=\theta(Z)-\theta(0)$ measures the twist of this flow [see Fig. 1]. In this example, the essence of the concept of monodromy resides in the multivaluedness of the function $\Theta(H, K)$, which exhibits a $2 \pi$ discontinuity along a loop surrounding the singular point, while $Z$ has no variation. During the relaxation process, the spatiotemporal system converges towards a stationary state that depends on the length $L$ and on the boundary conditions. Recalling that the stationary state converges toward the singular torus as $L \rightarrow \infty$ [5], it can be shown numerically that $L / Z \rightarrow 1$ for $L$ sufficiently large. In addition, using the generating function $F=$ $\left(I_{u}-I_{v}\right) \theta+I_{v} \psi$ where the angle $\psi$ is conjugate to the momentum $J=I_{v}$, one obtains that $\phi_{u}=\theta$ and $\phi_{v}=$ $\psi-\theta$. This yields a relation between the rotation number and the phase $\phi_{u}$ of the form $\phi_{u}(L)-\phi_{u}(0) \simeq \Theta$ when $L \simeq Z$. This explains the topological nature of the phasedefect formation discussed in Fig. 2. In particular, the $2 \pi$ phase shift of the wave is due to the $2 \pi$ discontinuity of $\Theta$, which thus represents a signature of the nontrivial monodromy of the system.

The linear dependence of $\phi_{u}(L)-\phi_{u}(0)$ vs $n$ reported in Fig. 2(b) can be explained through the analysis of the evolution of $\Theta$ with respect to the curvilinear coordinate along the loop, $s=\tan ^{-1}(H / \kappa K)$. In the neighborhood of the singular point $(h=0, k=0)$ where nonlinear cubic terms of Eq. (1) can be neglected, it can be shown that $\Theta(s)=\int_{0}^{Z(s)} \dot{\theta}(t) d t=s \quad$ where $\quad \dot{\theta}=\kappa \frac{H}{2 I_{u}} \quad$ [11]. This
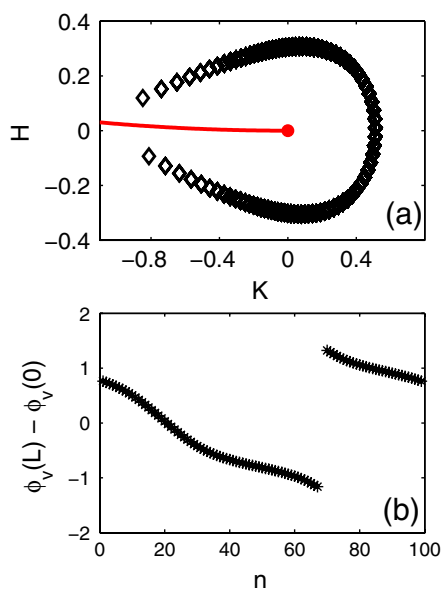

FIG. 5 (color online). (a) Zoom of the energy-momentum diagram near $H=K=0$ for the model (3). The grey (red) line denotes the singular curled tori ended by a singular pinched torus. The diamonds $(\diamond)$ denote the numerical loop. (b) Corresponding evolution of $\phi_{v}(L)-\phi_{v}(0)$ vs $n$ : Contrary to Fig. 2(b), the wave exhibits a $\pi$-phase-shift discontinuity. Parameters are $\varepsilon=0.1, \kappa=2$, and $\tau=100$. 
topological property also characterizes the PDE system, since this linear behavior has been observed numerically beyond the adiabatic regime [see Fig. 2(b)].

Extension to fractional monodromy.-This study may be generalized to nonstandard forms of Hamiltonian monodromy, such as fractional monodromy [8]. We illustrate this by considering the degenerate configuration of the resonant three-wave interaction [12],

$$
\begin{aligned}
& i\left(\frac{\partial u}{\partial t}+\frac{\partial u}{\partial z}\right)=-i v^{2}+\varepsilon u\left(|u|^{2}+\kappa|v|^{2}\right), \\
& i\left(\frac{\partial v}{\partial t}-\frac{\partial v}{\partial z}\right)=2 i u v^{*}+\varepsilon v\left(|v|^{2}+\kappa|u|^{2}\right) .
\end{aligned}
$$

where $\varepsilon$ and $\kappa$ are two real parameters. The corresponding stationary system is associated to an integrable Hamiltonian $H$ defined by

$$
H=-2 I_{v} \sqrt{2 I_{u}} \sin \left(\phi_{u}+2 \phi_{v}\right)+\varepsilon\left(I_{u}^{2}+I_{v}^{2}\right)+2 \varepsilon \kappa I_{u} I_{v}
$$

where $u=\sqrt{2 I_{u}} e^{i \phi_{u}}$ and $v=\sqrt{2 I_{v}} e^{-i \phi_{v}}$, and $K=I_{v}-$ $2 I_{u}$. This system admits a nontrivial fractional monodromy [8]. The corresponding energy-momentum diagram is reported in Fig. 5(a), together with a loop surrounding the singular pinched torus at $H=K=0$ and crossing the singular red line of curled tori. We followed this loop numerically by integrating Eq. (3). As illustrated in Fig. 5(b), the wave $v$ acquires a $\pi$ phase-shift discontinuity over the loop, in contrast with the $2 \pi$ discontinuity discussed above through Fig. 2(b). This is a consequence of the existence of a fractional monodromy in the system (3). We remark that, because of this fractional $\pi$ phase shift, the initial and final states of $v$ in the loop are different, although their corresponding coordinates in the energymomentum diagram coincide.

Conclusion and experimental perspectives.-We point out that the numerical simulations reported in Figs. 2 and 3 refer to a realistic experimental configuration that may be implemented in the context of nonlinear optics with currently available technology [9]. The nonlinear medium refers to a piece of Bragg-grating optical fiber, which is pumped at both ends by two optical waves whose frequencies match the Bragg frequency and whose amplitudes and phases may be modulated appropriately so as to describe loops in the energy-momentum diagram.

In summary, this work can be viewed as a new step in the understanding of the influence of the singularities of the stationary system on the spatiotemporal dynamics governed by PDEs. In this framework, another open question is the analysis of the relationship between these singularities and the soliton solutions of the PDEs in the corresponding infinitely extended medium.

The authors are grateful to K. Efstathiou, D. A. Sadovskii, and B. I. Zhilinskii for fruitful discussion.

*dominique.sugny@u-bourgogne.fr

[1] A. Bohm, A. Mostafazadeh, H. Koizumi, Q. Niu, and J. Zwanziger, The Geometric Phase in Quantum Systems (Springer, Berlin 2003).

[2] R.H. Cushman and L.M. Bates, Global Aspects of Classical Integrable Systems (Birkhäuser, Berlin, 1997); J. J. Duistermaat, Comm. Pure Appl. Math. 33, 687 (1980).

[3] R. H. Cushman and J. J. Duistermaat, Bull. Am. Math. Soc. 19, 475 (1988); S. Vu Ngoc, Commun. Math. Phys. 203, 465 (1999).

[4] K. Efstathiou and D. A. Sadovskií, Rev. Mod. Phys. 82, 2099 (2010); R. H. Cushman et al., Phys. Rev. Lett. 93, 024302 (2004); E. Assémat, K. Efstathiou, M. Joyeux, and D. Sugny, Phys. Rev. Lett. 104, 113002 (2010); H. R. Dullin and H. Waalkens, Phys. Rev. Lett. 101, 070405 (2008); N. J. Fitch et al., Phys. Rev. Lett. 103, 034301 (2009).

[5] D. Sugny, A. Picozzi, S. Lagrange, and H. R. Jauslin, Phys. Rev. Lett. 103, 034102 (2009).

[6] S. Lagrange, D. Sugny, A. Picozzi, and H. R. Jauslin, Phys. Rev. E 81, 016202 (2010); E. Assémat, A. Picozzi, H. R. Jauslin, and D. Sugny, Opt. Lett. 35, 2025 (2010).

[7] J. B. Delos, G. Dhont, D. A. Sadovskií, and B. I. Zhilinkií, Ann. Phys. (N.Y.) 324, 1953 (2009); J. B. Delos, G. Dhont, D. A. Sadovskií, and B. I. Zhilinskií, Europhys. Lett. 83, 24003 (2008).

[8] N. N. Nekhoroshev, D. A. Sadovskií and B. I. Zhilinskií, Ann. Inst. Henri Poincaré 7, 1099 (2006); D. Sugny et al., J. Math. Phys. (N.Y.) 49, 042701 (2008).

[9] Y.S. Kivshar and G. P. Agrawal, Optical Solitons: From Fibers to Photonics Crystals (Academic Press, San Diego, 2003).

[10] O. Morsch and M. Oberthaler, Rev. Mod. Phys. 78, 179 (2006); C. Conti and S. Trillo, Phys. Rev. Lett. 92, 120404 (2004).

[11] H. R. Dullin and S. Vu Ngoc, Nonlinearity 17, 1777 (2004); K. Efstathiou, Metamorphoses of Hamiltonian Systems with Symmetry, Lecture Notes in Mathematics (Springer-Verlag, Heidelberg, 2005).

[12] D. J. Kaup, A. Reiman, and A. Bers, Rev. Mod. Phys. 51, 275 (1979). 